\title{
Markers of kidney function in the elderly in relation to the new CKD-EPI formula for estimation of glomerular filtration rate
}

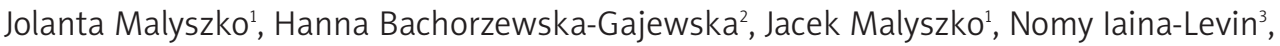 \\ Grazyna Kobus ${ }^{4}$, Slawomir Dobrzycki²
}

1Department of Nephrology and Transplantology, Medical University of Bialystok, Poland
2Department of Invasive Cardiology, Medical University of Bialystok, Poland
3Department of Nephrology and Hypertension, The Chaim Sheba Medical Center,
Tel-Hashomer, Israel
4Department of Clinical Medicine, Medical University of Bialystok, Poland

Submitted: 22 July 2010

Accepted: 9 November 2010

Arch Med Sci 2011; 7, 4: 658-664

DOI: 10.5114/aoms.2011.24136

Copyright @ 2011 Termedia \& Banach

\begin{abstract}
Introduction: Neutrophil gelatinase-associated lipocalin (NGAL) has been recently proved useful in the quantitation of chronic kidney disease (CKD). The aim of the study was to assess prevalence of CKD according to the Modification of Diet in Renal Disease (MDRD), Cockcroft-Gault, and the Chronic Kidney Disease Epidemiology Collaboration (CKD-EPI) formulae in 412 patients with normal serum creatinine and markers of kidney function/injury such as NGAL, cystatin C, and kidney injury molecule-1 (KIM-1) in these patients in relation to age (below and over 65 years).

Material and methods: Material and methods: We included in the study 1005 patients with coronary artery disease and normal serum creatinine. However, markers of kidney function/injury were assessed in 412 patients. The NGAL, cystatin $\mathrm{C}$ and KIM-1, were assessed using commercially available kits.

Results: Patients over 65 years had significantly lower estimated glomerular filtration rate (eGFR) than their younger counterparts despite identical creatinine. They also had significantly lower haematocrit, despite similar $\mathrm{Hb}$, lower platelet count, higher serum fibrinogen, higher systolic (SBP) and lower diastolic blood pressure, higher serum NGAL and cystatin C, but similar urinary NGAL and KIM-1. Serum NGAL correlated with age, haematocrit, leukocyte, platelet and erythrocyte count, eGFR, creatinine, fasting glucose, $\mathrm{HbA}_{1 c}$, fibrinogen, SBP, and diabetes duration. In multiple regression analysis kidney function (eGFR, creatinine clearance or creatinine), cystatin C and SBP were predictors of serum NGAL.

Conclusions: In our study we found a very high prevalence of CKD up to $61 \%$ in elderly patients with coronary artery disease and normal serum creatinine. Neutrophil gelatinase-associated lipocalin could be a sensitive marker of kidney function, particularly in elderly patients with another risk factor for kidney damage, i.e. hypertension.
\end{abstract}

Key words: elderly, neutrophil gelatinase-associated lipocalin, cystatin C, glomerular filtration rate, Chronic Kidney Disease Epidemiology Collaboration, hypertension, coronary artery disease.

\section{Introduction}

Prevalence of chronic kidney disease (CKD) is about $10-16 \%$, mainly in the elderly [1]. According to Kidney Disease Outcomes Quality Initiative

\author{
Corresponding author: \\ Prof. Jolanta Malyszko MD, \\ FASN \\ Department of Nephrology \\ and Transplantology \\ Medical University \\ of Bialystok \\ 14 Zurawia \\ 15-540 Bialystok, Poland \\ E-mail: jolmal@poczta.onet.pl
}


(K/DOQI) glomerular filtration rate (GFR) below 60 $\mathrm{ml} / \mathrm{min}$ is associated with increased risk of cardiovascular morbidity and mortality [2]. Kurtal et al. [3] pointed out that estimated glomerular filtration rate (eGFR) was better in predicting kidney function than creatinine; however, it was still an ideal assessment of renal function, particularly in the elderly. They did acknowledge that in the elderly, eGFR below $45 \mathrm{ml} / \mathrm{min}$ indicated a marked or severe decline in GFR, even without creatinine calibration. Moreover, in the very old population $>80$ years of age performance of both equations (Modification of Diet in Renal Disease [MDRD] and Cockcroft-Gault) has not yet been extensively studied against the gold standard measurement of GFR. In Third National Health and Nutrition Examination Survey (NHANES III) eGFR (MDRD) below $60 \mathrm{ml} / \mathrm{min}$ was found in $11 \%$ of subjects over 65 years without concomitant diabetes and hypertension, while only in $0.1 \%$ and $1.2 \%$ of subjects aged $20-39$ and $40-59$ years, respectively, was MDRD below $60 \mathrm{ml} / \mathrm{min}$ [1]. In a Japanese population of almost 100000 subject from Okinawa, in more than $80 \%$ of patients over 70 years creatinine clearance was below 60 $\mathrm{ml} / \mathrm{min}$ [4]. Due to different formulae used (MDRD, Cockcroft-Gault) results are not identical or even similar in the same age groups studied $[3,5,6]$. The Cockcroft-Gault equation provided higher estimates at younger ages, and lower estimates at older ages (i.e. greater than 70 years) than those obtained with the simplified MDRD formula [7]. It should be mentioned that age over 65 years is usually, but not always, associated with lowered GFR $[8,9]$. This may be due to the fact that there are many other comorbidities which may influence GFR $[10,11]$.

Estimation of GFR is used clinically to assess the degree of kidney impairment and to follow the course of the disease. The GFR is equal to the sum of the filtration rates in all of the functioning nephrons, giving a rough measure of their number. Glomerular filtration rate cannot be measured directly, but could be estimated from the urinary clearance of an ideal filtration marker or gold standard - inulin. The amount of inulin filtered at the glomerulus is equal to the amount excreted in the urine, which can be measured. However, inulin is in short supply, expensive, and difficult to assay. In addition, the classic protocol for measuring inulin clearance is full of drawbacks: continuous intravenous infusion, multiple blood samples, and bladder catheterization. Furthermore, various less cumbersome methods for measuring clearance are available such as alternative filtration markers (iothalamate, iohexol, DTPA, or EDTA), bolus administration of the marker, spontaneous bladder emptying, etc [12-14]. These methods are simpler, but all have disadvantages limiting their application in clinical practice and affecting the interpretation of research studies.

Since creatinine is also an unreliable parameter describing kidney function and true GFR is very rarely assessed, due to the costly, cumbersome and time-consuming procedure, the search for a new and sensitive marker of kidney function is under way. Neutrophil gelatinase-associated lipocalin (NGAL) has recently been proved useful in the quantitation of chronic kidney disease $[15,16]$. The ability to identify CKD may allow early implementation of treatments that could arrest or delay the progression of renal damage, enable effective treatment of its complications, and reduce the risk of drug-induced nephrotoxicity. Therefore, the aim of the study was to assess prevalence of chronic kidney disease using estimated GFR/creatinine clearance according to different formulae in 412 patients with normal serum creatinine undergoing percutaneous coronary interventions in relation to age (below and over 65 years) as well as markers of kidney function/injury such as NGAL, kidney injury molecule-1 (KIM-1) and cystatin C in this population.

\section{Material and methods}

We included in the study 1005 patients with coronary artery disease (confirmed angiographically, class II/III CCS) and normal serum creatinine (below $1.2 \mathrm{mg} / \mathrm{dl}$, the upper normal range in the central laboratory). The patients were recruited prospectively for percutaneous coronary intervention $(\mathrm{PCl})$ from the Invasive Cardiology Department. All patients were informed about the aim of the study and gave their informed consent. We excluded patients with pre-existing CKD, on the basis of elevated serum creatinine and/or history of kidney disease, i.e. proteinuria, erythrocyturia, etc. The study was approved by the Medical University Ethic Committee. Data were obtained from hospital clinical records.

We evaluated the patients' basal clinical variables: demographic and risk factors, family history in regard to coronary artery disease, history of myocardial infarction, serum creatinine on admission, lipids, glucose, blood pressure, medications. Serum creatinine was measured by the standard laboratory method (Jaffe) in one central laboratory. Haemoglobin, albumin, cholesterol and serum creatinine were analysed by the standard laboratory methods in the central laboratory at the University Hospital. We assessed kidney function according to the simplified MDRD formula [17] and the Cockcroft-Gault formula [18]. Since recently a new estimating equation for GFR, the Chronic Kidney Disease Epidemiology Collaboration (CKDEPI) equation, was developed [19], we also 
estimated GFR using this new formula. Staging of CKD was defined according to DOQI guidelines [1]. The NGAL was evaluated in serum using commercially available ELISA from BIOPORTO (Gentofte, Denmark). Cystatin C was estimated using a commercially available assay from BioVendor, Czech Republic. Kidney injury molecule-1 was assessed using commercially available kits from uscnlife, Shanghai, China. All tests were performed according to the manufacturer's instructions by the same person.

Data obtained were analysed using Statistica 7.0 computer software (Tulsa, OK). Data were expressed as means \pm SD or $\%$. The examination of the distribution normality of variables was done using the Shapiro-Wilk W test. Comparisons between groups were done by ANOVA or $\chi^{2}$ test as appropriate. Correlations between NGAL and other variables were evaluated by Pearson's or Spearman's test as appropriate. Values of $p<0.05$ were taken as statistically significant. Multiple regression analysis was used to determine independent factors affecting the dependent variable. Factors showing a linear correlation with NGAL $(p<0.1)$ were included in the analysis.

\section{Results}

In the group of patients below 65 years with normal serum creatinine according to the Cockcroft-
Gault formula, stage 3 CKD was found in $32 \%$, according to MDRD in $6 \%$, and according to CKDEPI in $6 \%$ of patients below 65 years of age. Normal eGFR, i.e. $90-120 \mathrm{ml} / \mathrm{min}$, was found in $21 \%$ (Cockcroft-Gault formula), 40\% (MDRD) and 39\% (CKD-EPI).

In the group of elderly patients (over 65 years) with normal serum creatinine according to CockcroftGault, stage 3 CKD, i.e. GFR 30-59 ml/min, was found in $61 \%$, and stage 4 CKD (GFR $15-29 \mathrm{ml} / \mathrm{min}$ ) in $4 \%$ patients. Normal eGFR was found in $7 \%$ of patients older than 65 years. According to MDRD, stage 3 was diagnosed in 19\%, while normal eGFR was diagnosed in $21 \%$ of patients older than 65 years. According to CKD-EPI, stage 3 was diagnosed in $19 \%$ of patients over 65 years of age, stage 4 in 1\%, and normal eGFR in $21 \%$ of this population. They had significantly lower haematocrit, despite similar $\mathrm{Hb}$, lower platelet count, higher serum fibrinogen, higher systolic and lower diastolic blood pressure (Table I). Patients over 65 years of age had significantly lower eGFR (MDRD, CKD-EPI, CockcroftGault formulae) than their younger counterparts despite identical serum creatinine and significantly higher serum NGAL and cystatin C, but similar urinary NGAL (Table II). Serum NGAL correlated with age $(r=-0.27, p<0.001)$ (Figure 1$)$, haematocrit $(r=-0.24, p<0.001)$, haemoglobin $(r=-0.12$, $p<0.01)$, leukocyte $(r=-0.15, p<0.01)$, platelet

Table I. Clinical characteristics of the 412 patients with normal serum creatinine undergoing primary coronary interventions in regard to age

\begin{tabular}{|c|c|c|c|}
\hline Parameters & Age $<65$ years & Age $>65$ years & Value of $p$ \\
\hline Age [years] & $56.22 \pm 6.02$ & $77.74 \pm 4.71$ & $<0.001$ \\
\hline $\mathrm{BMI}\left[\mathrm{kg} / \mathrm{m}^{2}\right]$ & $29.53 \pm 4.72$ & $28.87 \pm 4.52$ & $>0.05$ \\
\hline $\operatorname{RBC}\left[\times 10^{12} / \mathrm{I}\right]$ & $4.63 \pm 0.47$ & $4.89 \pm 0.51$ & $<0.01$ \\
\hline $\mathrm{Hb}[\mathrm{g} / \mathrm{dl}]$ & $13.98 \pm 1.32$ & $13.68 \pm 2.82$ & $>0.05$ \\
\hline Hct [\%] & $41.29 \pm 4.01$ & $39.97 \pm 4.18$ & $<0.001$ \\
\hline Platelets $\left[\times 10^{6} / 1\right]$ & $234.07 \pm 63.6$ & $215.38 \pm 58.13$ & $<0.001$ \\
\hline WBC $\left[\times 10^{6} / I\right]$ & $7.60 \pm 2.18$ & $7.42 \pm 2.6$ & $>0.05$ \\
\hline Total cholesterol [mg/dl] & $169.91 \pm 44.1$ & $162.21 \pm 39.8$ & $>0.05$ \\
\hline LDL cholesterol [mg/dl] & $98 \pm 36.6$ & $92.1 \pm 34.86$ & $>0.05$ \\
\hline HDL cholesterol [mg/dl] & $42.42 \pm 11.87$ & $42.9 \pm 12.3$ & $>0.05$ \\
\hline Triglycerides [mg/dl] & $152.8 \pm 111.18$ & $139.9 \pm 77.99$ & $>0.05$ \\
\hline Glucose [mg/dl] & $114.46 \pm 40.57$ & $112.7 \pm 41.66$ & $>0.05$ \\
\hline Insulin [mIU/l] & $15.77 \pm 29.86$ & $10.25 \pm 5.58$ & $>0.05$ \\
\hline $\mathrm{HbA}_{1 \mathrm{c}}[\%]$ & $6.36 \pm 1.11$ & $6.46 \pm 1.23$ & $>0.05$ \\
\hline Fibrinogen $[\mathrm{mg} / \mathrm{dl}]$ & $396.9 \pm 88.13$ & $428.67 \pm 91.04$ & $<0.001$ \\
\hline Systolic BP [mmHg] & $125.68 \pm 17.69$ & $132.3 \pm 19.81$ & $<0.001$ \\
\hline Diastolic BP [mmHg] & $75.96 \pm 11.05$ & $72.96 \pm 10.8$ & $<0.01$ \\
\hline EF [\%] & $47.79 \pm 11.15$ & $47.42 \pm 10.14$ & $>0.05$ \\
\hline
\end{tabular}


Table II. Kidney function of the 412 patients with normal serum creatinine undergoing primary coronary interventions in regard to age

\begin{tabular}{|lccc|}
\hline Parameters & Age $<65$ years & Age $>65$ years & Value of $p$ \\
\hline Creatinine $[\mathrm{mg} / \mathrm{dl}]$ & $1.05 \pm 0.61$ & $1.07 \pm 0.33$ & $>0.05$ \\
\hline MDRD formula $[\mathrm{ml} / \mathrm{min}]$ & $84.11 \pm 19.76$ & $71.18 \pm 20.21$ & $<0.001$ \\
\hline CKD-EPI formula $[\mathrm{ml} / \mathrm{min}]$ & $84.52 \pm 18.21$ & $67.58 \pm 18.41$ & $<0.001$ \\
\hline C-G formula $[\mathrm{ml} / \mathrm{min}]$ & $100.39 \pm 27.74$ & $67.91 \pm 21.48$ & $<0.001$ \\
\hline NGAL - serum $[\mathrm{mg} / \mathrm{ml}]$ & $60.51 \pm 27.11$ & $78.21 \pm 35.78$ & $<0.001$ \\
\hline NGAL - urine $[\mathrm{mg} / \mathrm{ml}]$ & $16.19 \pm 14.87$ & $17.01 \pm 21.76$ & $>0.05$ \\
\hline Cystatin C - serum $[\mathrm{mg} / \mathrm{l}]$ & $0.97 \pm 0.45$ & $1.20 \pm 0.43$ & $<0.001$ \\
\hline Urinary KIM-1 $[\mathrm{mg} / \mathrm{ml}]$ & $45.96 \pm 32.69$ & $50.97 \pm 46.54$ & $>0.05$ \\
\hline
\end{tabular}

$(r=0.11, p<0.05)$ and erythrocyte count $(r=-0.18$, $p<0.001)$, eGFR/estimated creatinine clearance (eCrCl) (MDRD, $r=-0.48, p<0.001)$, CKD-EPI $(r=-0.52, p<0.001$, Figure 2$)$, Cockcroft-Gault, $r=-0.41, p<0.001)$, serum creatinine $(r=0.49$, $p<0.001)$, fasting glucose $(0.18, p<0.01), \mathrm{HbA}_{1 \mathrm{c}}$ $(r=0.13, p<0.05)$, fibrinogen $(r=0.35, p<0.001)$, NYHA class $(r=0.28, p<0.001)$, SBP $(r=0.22$, $p<0.01)$, diabetes duration $(r=0.21, p<0.05)$, urinary NGAL $(r=0.41, p<0.001)$, serum cystatin C ( $r=0.58, p<0.001$, Figure 3$)$, and urinary KIM-1 $(r=0.13, p<0.05)$.

In the model of multiple regression analysis parameters which correlated or tended to correlate with NGAL $(p<0.1)$ were included (age, haematocrit, leukocyte count, platelet count, eGFR/eCrCl or serum creatinine, fasting glucose/ $/ \mathrm{HbA}_{1 \mathrm{c}}$, fibrinogen, NYHA class, cystatin C, duration of diabetes, duration of hypertension, urinary NGAL, urinary $\mathrm{KIM}-1$, systolic blood pressure). In multiple regression analysis kidney function (either eGFR $\beta$ value was -0.38 , $p=0.0003$, or serum creatinine $\beta$ value was 0.33 , $p=0.003)$, cystatin $C$ ( $\beta$ value was 0.23 , $p=0.017)$ and systolic blood pressure $(\beta$ value $0.18, p=0.05$ ) were predictors of serum NGAL,



Figure 1. Corelation between age and serum NGAL explaining $77 \%$ of $\mathrm{NGAL}$ variation, $\mathrm{F}=9.71$, $\mathrm{SE}=22.57$.

Impact of diabetes, hypertension and gender of elderly patients on serum neutrophil gelatinase-associated lipocalin

Normotensive elderly patients had significantly lower NGAL $(106 \pm 22.8 \mathrm{ng} / \mathrm{ml}$ vs. $129.6 \pm 47.0 \mathrm{ng} / \mathrm{ml}$, $p<0.05)$ than hypertensives. Despite similar serum creatinine levels, eGFR/eCrCl was significantly higher in normotensives $(p<0.05)$ than in hypertensives. Serum NGAL was significantly higher in diabetics than in non-diabetics $(132.12 \pm 50.21 \mathrm{ng} / \mathrm{ml}$ vs. 94.56 $\pm 55.21 \mathrm{ng} / \mathrm{ml}, p<0.05)$, whereas creatinine did not differ significantly between these groups. Females had lower eGFR (MDRD and CKD-EPI formulae) and $\mathrm{eCrCl}$ relative to males (MDRD $54.89 \pm 21.61 \mathrm{ml} / \mathrm{min}$ vs. $73.21 \pm 20.98 \mathrm{ml} / \mathrm{min}, p<0.01$, Cockcroft-Gault $48.11 \pm 18.79 \mathrm{ml} / \mathrm{min}$ Vs. $61.35 \pm 17.87 \mathrm{ml} / \mathrm{min}$, $p<0.05$, CKD-EPI $54.76 \mathrm{ml} / \mathrm{min} \pm 20.45$ vs. 71.69 $\pm 18.19 \mathrm{ml} / \mathrm{min}, p<0.01)$.

Patients over 65 years of age had statistically significantly lower diastolic blood pressure, lower body mass index (BMI), higher serum urea, and lower eGFR/creatinine clearance (all formulae)

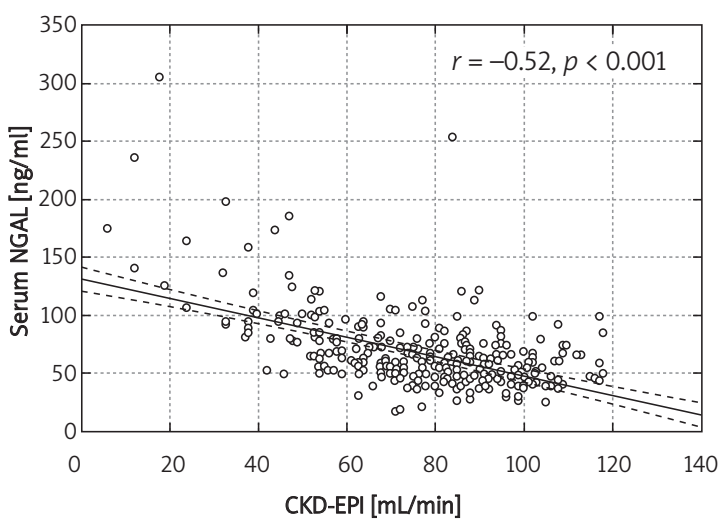

Figure 2. Corelation between serum NGAL and eGFR (CKD-EPI) 


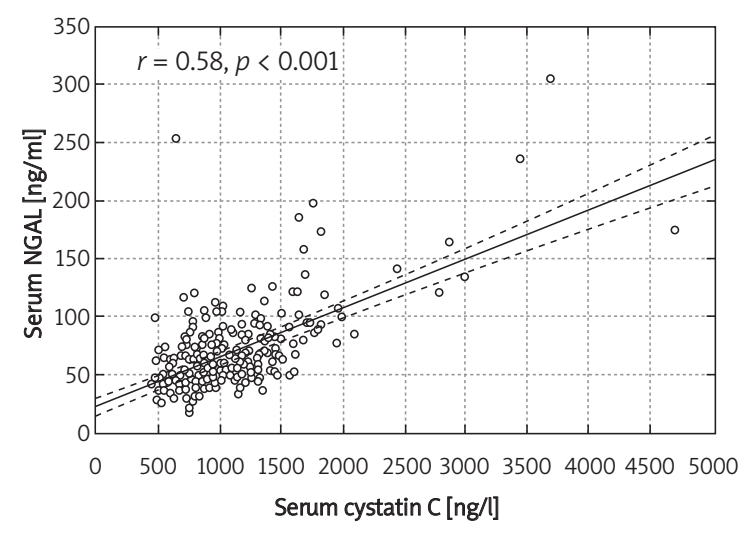

Figure 3. Corelation between cystatin CNGAL and serum NGAL

despite similar serum creatinine. The prevalence of CKD, based on the National Kidney Foundation Kidney Disease Outcome Quality Initiative (NKF/DOQI) guidelines, was higher in the elderly. However, prevalence of diabetes $(20 \%$ vs. $30 \%$, $p<0.05)$, dyslipidaemia ( $28 \%$ vs. $63 \%, p<0.001$ ), positive family history of cardiovascular diseases ( $91 \%$ vs. $42 \%, p<0.001)$, past myocardial infarction (14\% vs. $20 \%, p<0.05)$ and smoking ( $24 \%$ vs. $84 \%$, $p<0.001)$ was significantly lower than in their younger counterparts (below 65 years), while hypertension was more prevalent in older patients (higher systolic blood pressure with lower diastolic blood pressure) (73\% vs. $59 \%, p<0.01)$.

\section{Discussion}

Kidney function declines with years; however, the important problem remains the assessment of kidney function in the elderly. Unfortunately, creatinine is an unreliable parameter describing kidney function [20]. First, using serum creatinine to estimate true renal function has well-recognized inaccuracies and limitations [20]. A marked reduction in GFR can be present before it is reflected in a rise in serum creatinine (up to 50\% of kidney function has already been lost before creatinine might change). Serum creatinine levels can vary widely with age, gender, muscle mass, muscle metabolism, medication and hydration status. In the Baltimore Longitudinal Study, 254 healthy participants (without CKD, hypertension, not treated with diuretics) had periodic assessment of creatinine clearance during the period 1958-1981 [21]. Mean decrease in creatinine clearance was $0.75 \mathrm{ml} / \mathrm{min} / \mathrm{year}$, and was greater in patients who developed hypertension later during observation. In 1/3 there was no fall in creatinine clearance. In all the formulae used to estimate GFR, including the recent one i.e. CKD-EPI, age is included; however, so far there are no data on validation of them in the elderly population. Within the $5^{\text {th }}$ and $95^{\text {th }}$ percentile for age, both
Cockcroft-Gault and MDRD formulae provide similar measurements, which were consistent with agespecific historic inulin clearance values [1]. The Cockcroft-Gault equation provided higher estimates at younger ages, and lower estimates at older ages (i.e. greater than 70 years) than those obtained with the simplified MDRD formula [7]. Stevens et al. [22] reported in a meta-analysis on 5504 patients that, for those with an initial estimated GFR of $60 \mathrm{ml} / \mathrm{min}$ per $1.73 \mathrm{~m}^{2}$ or greater, the median difference between measured and estimated GFR was $8.3 \mathrm{ml} / \mathrm{min}$ per $1.73 \mathrm{~m}^{2}$, which represents an underestimation of GFR. This is due to the fact that the MDRD formula was developed mainly in the population with kidney disease and true GFR below $60 \mathrm{ml} / \mathrm{min}$. Compared to the Cockcroft-Gault equation however, the MDRD study equation that is recalibrated for use with standardized creatinine values provides more accurate estimates of the glomerular filtration rate [23].

In a recent issue of Gerontology, Kurtal et al. [2] reported that undiagnosed CKD was common in the elderly. Moreover, in this study in about $52 \%$ of the prescribed drugs and about $65 \%$ of all prescribed drugs were those that needed dose adjustment or should be avoided in renal impairment. Since interventional cardiologists are being asked to perform $\mathrm{PCl}$ on increasing numbers of elderly patients with significant co-morbidities, such as diabetes and/or renal insufficiency, contrast nephropathy is a potentially serious complication of $\mathrm{PCl}[24,25]$. Other risk factors also should be taken into accounts as contributors of $\mathrm{PCl}$ complications and adverse outcomes [26-29].

In our study, we found that serum NGAL correlated with kidney function/dysfunction in the population studied. The strongest predictor of serum NGAL was eGFR, indicating utility of NGAL measurements in these patients. It has been recently reported that $\mathrm{NGAL}$ could represent a marker of renal function in chronic kidney disease in children [30] and adults [12]. Neutrophil gelatinase-associated lipocalin is synthesized systemically in response to kidney damage followed by glomerular filtration and tubular uptake, and could be produced locally by injured tubules. As a small molecule NGAL is freely filtered by the glomerulus, then is largely absorbed in the proximal tubules by efficient megalin-dependent endocytosis [31]. Mori et al. [32] showed that a systemic injection of labelled NGAL resulted in accumulation of NGAL in the proximal tubules without concomitant appearance in urine. Thus, NGAL excretion in urine, in the situation of associated proximal tubule injury, is likely to be due to NGAL reabsorption and/or enhanced de novo NGAL synthesis. In our study we did not find statistical differences in urinary NGAL between patients older 
vs. younger than 65 years. Our previous study demonstrated that hypertensive patients had significantly higher serum NGAL than their normotensive counterparts [33]. Our present study is in line with this finding. In addition, in our recent studies on heart transplant recipients, we found that the prevalence of CKD in the elderly population was as high as $81 \%$ (MDRD, CKD-EPI, creatinine clearance) to $100 \%$ (Cockcroft-Gault formula), whereas in the younger population of heart transplant recipients the prevalence of CKD ranged from $56.3 \%$ to $77.5 \%$ [34]. Moreover, elderly heart recipients had significantly higher cystatin $\mathrm{C}$, serum NGAL and lower eGFR than their younger counterparts, despite not having statistically different serum creatinine. Neutrophil gelatinaseassociated lipocalin was related to markers of kidney function (eGFR, creatinine, cystatin C) in heart transplant recipients [34, 35]. However, we should bear in mind that NGAL is also found in neutrophils.

The best predictor of NGAL was eGFR; thus we should take into account the usefulness of NGAL estimation in clinical practice. In the perspective of the development of urinary [36] or whole blood/plasma assays [37] for NGAL estimation, a new tool for assessment of kidney function would be available. The assay for the whole blood or plasma is easy with quantitative results available in 15 min and requires only microlitre sample [37]. Urinary assay requires only $150 \mu \mathrm{l}$ of urine and the results are available within $35 \mathrm{~min}$ [36].

The strengths of our study are simultaneous measurement of urinary, serum NGAL, urinary KIM-1 and serum cystatin C, age- and sex-matching for the populations over and below 65 years of age, and use of the novel formula CKD-EPI, which better determines eGFR in patients over $60 \mathrm{ml} / \mathrm{min}$ and had validation for diabetes, age and transplantation (although transplantation was not the case in this setting). The limitations are that this study is singlecentre, cross-sectional, and on patients with normal serum creatinine. Lack of GFR measurement may also be a limitation; however, this procedure is costly, cumbersome and generally not used for clinical purposes, only for research.

Chronic kidney disease is an under-recognized problem in geriatrics and eGFR should be automatically reported, not only in geriatric units, but in every unit, since our patients are getting older, have more concomitant diseases, receive more and more drugs and undergo more and more potentially nephrotoxic invasive procedures such as $\mathrm{PCl}$ or computed tomography with contrast administration. Neutrophil gelatinase-associated lipocalin should be investigated as a potential early and sensitive marker of kidney impairment/injury. Moreover, due to its sensitivity, NGAL may uncover nephrotoxicity of new medications or procedures in this vulnerable population of elderly patients.

\section{References}

1. Coresh J, Astor BC, Greene T, Eknoyan G, Levey AS. Prevalence of chronic kidney disease and decreased kidney function in the adult US population: Third National Health and Nutrition Examination survey. Am J Kidney Dis 2003; 41: 1-12.

2. Kurtal H, Schwenger V, Azzaro M, et al. Clinical value of automatic reporting of estimated glomerular filtration rate in geriatrics. Gerontology 2009; 55: 288-95.

3. National Kidney Foundation K/DOQI: Clinical Practice Guidelines for chronic kidney disease: evaluation, classification, and stratification. Am J Kidney Dis 2002; 39: S1-266.

4. Iseki K, Kinjo K, Iseki C, Takishita S. Relationship between predicted creatinine clearance and proteinuria and the risk of developing ESRD in Okinawa, Japan. Am J Kidney Dis 2004; 44: 806-14.

5. Froissart M, Rossert J, Jacquot C, Paillard M, Houillier P. Predictive performance of the MDRD and Cockcroft-Gault equations for estimating renal function. J Am Soc Nephrol 2005; 16: 763-73.

6. Verhave JC, Fesler P, Ribstein J, du Cailar G, Mimran A. Estimation of renal function in subjects with normal serum creatinine levels: influence of age and body mass index. Am J Kidney Dis 2005; 46: 233-41.

7. Collins AJ, Li S, Gilbertson DT, Liu J, Chen SC, Herzog CA. Chronic kidney disease and cardiovascular disease in the Medicare population. Kidney Int Suppl 2003; 87: S24-31.

8. Fliser D, Franek E, Joest M. Renal function in the elderly: Impact of hypertension and cardiac function. Kidney Int 1997; 51: 1196-204.

9. Feinfeld DA, Keller S, Somer B, et al. Serum creatinine and blood urea nitrogen over a six-year period in the very old. Geriatr Nephrol Urol 1998; 8: 131-5.

10. Bachorzewska-Gajewska H, Malyszko J, Malyszko JS, Musial WJ, Dobrzycki S. Undiagnosed renal impairment in patients with and without diabetes with normal serum creatinine undergoing percutaneous coronary intervention. Nephrology 2006; 11: 549-54.

11. Bachorzewska-Gajewska H, Malyszko J, Malyszko JS, Dobrzycki S, Sobkowicz B, Musial W. Estimation of glomerular filtration rate in patients with normal serum creatinine undergoing primary $\mathrm{PCl}$ : is it really normal? Nephrol Dial Transplant 2006; 21: 1736-9.

12. Rahn KH, Heidenreich S, Bruckner D. How to assess glomerular function and damage in humans. J Hypertens 1999; 17: 309-17.

13. Levey AS. Measurement of renal function in chronic renal disease. Kidney Int 1990; 38: 167-84.

14. Brandstrom E, Grzegorczyk A, Jacobsson L, Friberg P, Lindahl A, Aurell M. GFR measurement with iohexol and 51Cr-EDTA. A comparison of the two favoured GFR markers in Europe. Nephrol Dial Transplant 1998; 13: 1176-82.

15. Malyszko J, Malyszko JS, Bachorzewska-Gajewska H, Poniatowski B, Dobrzycki S, Mysliwiec M. Neutrophil gelatinase-associated lipocalin is a new and sensitive marker of kidney function in chronic kidney disease patients and renal allograft recipients. Transplant Proc 2009; 41: 158-61.

16. Bolignano D, Lacquaniti A, Coppolino G, et al. Neutrophil gelatinase-associated lipocalin (NGAL) and progression of chronic kidney disease. Clin J Am Soc Nephrol 2009; 4: 337-44. 
17. Modification of diet in Renal Disease Study Group. Creatinine filtration, secretion and excretion during progressive renal disease. Kidney Int 1989; 36: S73-80.

18. Cockcroft DW, Gault MH. Prediction of creatinine clearance from serum creatinine. Nephron 1976; 16: 31-41.

19. Levey AS, Stevens LA, Schmid CH, et al.; CKD-EPI (Chronic Kidney Disease Epidemiology Collaboration). A new equation to estimate glomerular filtration rate. Ann Intern Med 2009; 150: 604-12.

20. Bellomo R, Kellum JA, Ronco C. Defining acute renal failure: physiological principles. Intensive Care Med 2004, 30: 33-7.

21. Lindeman RD, Tobin J, Shock NW. Association between blood pressure and the rate of decline in renal function with age. Kidney Int 1984; 26: 861-8.

22. Stevens LA, Coresh J, Feldman HI, et al. Evaluation of the modification of diet in renal disease study equation in a large diverse population. J Am Soc Nephrol 2007; 18: 2749-57.

23. Stevens LA, Nolin TD, Richardson MM, et al. Comparison of drug dosing recommendations based on measured GFR and kidney function estimating equations. Am J Kidney Dis 2009; 54: 33-42.

24. Malyszko J, Bachorzewska-Gajewska H, Malyszko JS, Dobrzycki S. Prevalence of chronic kidney disease in elderly patients with normal serum creatinine levels undergoing percutaneous coronary interventions. Gerontology 2010; 56: 51-4.

25. Uyarel H, Cam N, Ergelen M, et al. Contrast-induced nephropathy in patients undergoing primary angioplasty for acute myocardial infarction: incidence, a simple risk score, and prognosis. Arch Med Sci 2009; 5: 550-8.

26. Khosravi AR, Kelishadi R, Poormoghadas M, Shirani S, Asgary S, Khosravi M. Preprocedural C-reactive protein predictive value in angiographic in-stent restenosis after coronary stent placement in patients with stable angina. Arch Med Sci 2009; 5: 166-71.

27. Tomaszuk-Kazberuk A, Sobkowicz B, Dobrzycki S, Lewczuk A, Musial W. Perfusion assessed by real-time contrast echocardiography correlates with clinical and echocardiographic parameters in patients with first STEM treated with $\mathrm{PCl}$ - 6-month follow-up. Arch Med Sci 2010; 6: 176-82.

28. Sethi A, Arora RR. Ambulatory blood pressure as a predictor of cardiovascular risk. Arch Med Sci 2009; 5: $3-9$

29. Katsiki N, Mikhailidis DP, Athyros VG, Hatzitolios Al, Karagiannis A, Banach M. Are we getting to lipid targets in real life? Arch Med Sci 2010; 6: 639-41.

30. Mitsnefes MM, Kathman TS, Mishra J, et al. Serum neutrophil gelatinase-associated lipocalin as a marker of renal function in children with chronic kidney disease. Pediatr Nephrol 2007; 22: 101-8.

31. Schmitt-Ott KM, Mori K, Li JY, et al. Dual action of neutrophil gelatinase-associated lipocalin. J Am Soc Nephrol 2007; 18: 407-13.

32. Mori K, Lee HT, Rapoport D, et al. Endocytic delivery of lipocalin-siderophore-iron complex rescues the kidney from ischemia-reperfusion injury. J Clin Invest 2005; 115: 610-21.

33. Malyszko J, Bachorzewska-Gajewska H, Malyszko JS, Pawlak K, Dobrzycki S. Serum neutrophil gelatinaseassociated lipocalin as a marker of renal function in hypertensive and normotensive patients with coronary artery disease. Nephrology (Carlton) 2008; 13: 153-6.

34. Przybylowski P, Malyszko J, Malyszko J. Kidney function assessed by eGFR, cystatin C and NGAL (neutrophil gelatinase-associated lipocalin) in relation to age in heart allograft recipients. Med Sci Monit 2010; 16: CR440-4.

35. Przybylowski P, Malyszko J, Malyszko JS. Serum neutrophi gelatinase associated lipocalin correlates with kidney function in heart allograft recipients. Transplant Proc 2010; 42: 1797-802.

36. Nickolas TL, O'Rourke MJ, Yang J, et al. Sensitivity and specificity of a single emergency department measurement of urinary neutrophil gelatinase-associated lipocalin for diagnosing acute kidney injury. Ann Intern Med 2008; 148: 810-9.

37. Dent CL, Ma Q, Dastrala S, et al. Plasma NGAL predicts acute kidney injury, morbidity and mortality after pediatric cardiac surgery: a prospective uncontrolled cohort study. Crit Care 2007; 11: R127. 